\title{
Development of an Adaptive Hybrid Technique to Mitigate Cross-Tier Interference in a Femto-Macro Heterogeneous Network
}

\author{
Magaji Suleiman \\ Electrical and Computer \\ Engineering Department, \\ Ahmadu Bello University, \\ Zaria, Nigeria
}

\author{
S. M. Sani \\ Electrical and Computer \\ Engineering Department, \\ Ahmadu Bello University Zaria, \\ Nigeria.
}

\author{
A. M. S. Tekanyi \\ Electrical and Computer \\ Engineering Department, \\ Ahmadu Bello University \\ Zaria, Nigeria.
}

\begin{abstract}
The deployment of small cell node such as femto-cell within macro area coverage has been considered a promising solution to provide better throughput and Quality of Experience (QoE) to users. However, co-channel deployment of femto-cell operating in a closed access mode (CSG) causes severe cross-tier interference. In this paper we have develop an adaptive Hybrid Power control and Time domain Technique (aHPTT) to mitigate cross-tier interference in HetNet. The hybrid technique was developed to handle limitations associated with using a single interference mitigation technique such as Power Control (PC) and Time Domain (TD). aHPTT integrate PC and TD to mitigate interference simultaneously depending on the position of the user in the network. The hybrid technique was implemented in a Graphical User Interface (GUI) using MATLAB R2013a. System level simulation was carried out to evaluate the performance of the hybrid technique in terms of user's throughput. The aHPTT was validated with the $3^{\text {rd }}$ Generation Partnership Project (3GPP) enhance Inter-cell Interference Coordination (eICIC) technique, results obtained showed that the aHPTT performs better than PC technique by $2.90 \%$ and TD technique by $82.60 \%$.
\end{abstract}

\section{General Terms}

Interference Management

\section{Keywords}

Interference, Power Control, ABS, aHPTT, eICIC, HetNet. Signal-to-Interference plus Noise Ratio (SINR).

\section{INTRODUCTION}

Improving the indoor coverage and providing high-data-rate services in a cost effective manner and at the same time enhancing capacity is one of the major challenges in Next Generation Network (NGN) such as the $3^{\text {rd }}$ Generation Partnership Project (3GPP) Long Term Evolution (LTE) network [1]. The demand for data is increasing exponentially and expected to grow up to 30.6 ExaByte (EB) per month by the year 2020, this is eight times the total traffic in 2015 [2]. Studies has shown that $70 \%$ of the total data traffic and $50 \%$ of the total voice traffic are generated indoors [3,4]. This makes it important for the operators to find the means of improving indoor coverage. One of the ways to improve indoor coverage is to reduce transmit-receive distance by bringing the transmitter and receiver closer to each other.
Femto-cell is considered a promising solution for the mobile operators to provide better quality of service $(\mathrm{QoS})$ for indoor users [5]. The advantages of deploying femto-cell is enormous in terms of technical and business point of view [1, 6]:

i. Femto-cell improve coverage and capacity due to short transmit-receive distance.

ii. Provides high data rate and call quality to it users.

iii. Improves macro cell efficiency and reliability by offloading traffic away from macro cell.

iv. Deployment of femto-cell is cost effective and selfoptimizing, therefore reducing the capital and operational expenditure (CAPEX, OPEX) of the network.

v. Femto-cell can easily be deployed by customers without any challenge; because it is a plug-and-play device with self-optimizing capability.

vi. Prolong the battery life of the user equipment since it require less energy to connect to a closer base station.

Deployed femto-cell can make use of the same or different carrier frequencies with the existing macro network. The use of different carrier frequency avoid the interference between macro- and femto-cell, but requires more bandwidth. Due to the fact that licensed spectrum is expensive and scarce, operators prefer to use the same carrier frequency (cochannel) to reduce spectrum requirement [7]. Although, cochannel deployment ensure larger bandwidth and efficient spectrum utilization for both macro- and femto-cell, the resulting cross-tier interference may prevent macro cell users within the coverage area of femtocells from having high Signal-to-Interference plus Noise Ratio (SINR) [8]. Femtocells are typically installed in a closed access mode [9] were only users enlisted in the closed subscriber group (CSG) can have access. Therefore in the downlink transmission, macrousers close to femto-cell operating in CSG mode experience severe interference from the femto-cell [9].

Interference is the major challenge in femto-cell deployment $[4,10]$. Therefore, to fully utilize the advantages of femto-cell deployment, it is important to mitigate the interference that comes along with it deployment. Frequency Domain (FD), Time Domain (TD) and Power Control (PC) were introduced by the $3^{\text {rd }}$ generation partnership project in it enhanced InterCell Interference Coordination (eICIC) technique to mitigate interference in LTE-A rel. 10 standards $[11,12]$. Several interference mitigation techniques in heterogeneous network 
has been proposed in the open literature. However, existing interference technique in a femto-macro heterogeneous network mitigate interference using a single eICIC technique [13] which mitigate interference at the expense of throughput. In [14] the authors proposed an adaptive power level setting scheme to mitigate the interference in femto-macro Heterogeneous Network (HetNet), the scheme considered penetration losses and adjustable positive factors to estimate the priority of femto-cell power operations. Authors in [15] proposed a macro assisted Home enhanced Node B (HeNB) power control scheme to mitigate interference resulting from femto cell. The technique adjust the transmit power of HeNB when it receive interference message from a macro user equipment experiencing interference. [16] proposed a distributive technique to determine victim user equipment protection from interference by almost blank sub-frame. The assumed that all pico UE are scheduled only during ABS, while the number of $\mathrm{ABS}$ to be created is determine by the rule of thumb. An optimized time domain resource partitioning was carried out to obtain ABS density and configuration. In [17] an orthogonal ABS scheme was proposed. The number of sub frame to be blanked was dependent on the number of victim users and normal users who are not interfered by the aggressor node. [18] solve the coupled problems of: (i) determining the amount of radio resources that macro cells should offer to pico-cells and (ii) determining the association rules that decide which user equipment should associate with pico cell. Cell Selection Biasing (CSB) was used to determine user association, while the amount of radio resources was determine using ABS. The two solutions where implemented in a joint manner. Authors in [7] proposed a simple approach to determine adaptively the most suitable value of the muting ratio as a function of the network traffic load based on suitable threshold values. In their model, femto cell was assume to be operating in open subscriber mode (OSG). ABS was used to mitigate the interference faced by users in the cell range expansion region (CRE). A novel technique which combine interference cancellation and interference avoidance to mitigate downlink interference in a femto-macro HetNet was developed in [19]. At the receiver side a downlink interference cancellation scheme was developed to reduce interference impact on users by optimizing their SINR. Interference avoidance techniques based on subframe blanking was used to mitigate interference at the aggressor cell. [8] proposed a femto power control strategy for mitigating the interference experience by macro cell users while preventing femto-cell throughput degradation. The proposed power control schemes make use of femto and macro user's context information in terms of positioning for setting the appropriate prioritization weights among the victim macro users and still maintained a high performance for femto cell users when compared to a conventional power control scheme.

It is evident from above that most work done on interference mitigation in HetNet is by either Power Control (PC) or time domain through the use of ABS. However, the use of a single eICIC technique, mitigate interference at the expense of reduced aggressor cell capacity. Power control in femto-cell mitigate the interference on victim users at the cell boundary of femto-cell by reducing its power but is limited when the victim user is very close to the femto-cell because some of the femto users can go out of coverage when the power is reduced. Time domain technique can effectively mitigate the interference of victim user closer to the femto-cell by creating blank sub-frames but decreases the throughput of the aggressor cell because data is not transmitted during almost blank sub-frame. Therefore an adaptive Hybrid Power-control and Time-domain technique (aHPTT) has been developed to mitigate the cross-tier interference in heterogeneous femtomacro network. The technique mitigate the interference of victim users depending on their position in the network with insignificant reduction in the aggressor capacity.

\section{FEMTO-MACRO NETWORK}

The deployment of femto-cells in an existing all macro network can introduce cross-tier Inter- Cell Interference (ICI) to the network. The restricted access control associated with femto-cell when deployed in closed access mode may lead to strong interference scenario in both uplink and downlink [11, 20]. Femto-cells are not connected directly to the core network of the operator, therefore only limited backhaul signaling for interference coordination is possible. Femto-cell are also deployed in ad-hoc manner without operator supervision [21]. This means that proper operation of femtocell will highly depend on their self-organizing features, sensing and continuous monitoring of the radio environment in order to adaptively mitigate or avoid interference [22]. When a macro user especially at the boundary of a macroand femto-cell comes within the strong signal coverage of a femto-cell operating in Closed Subscriber Group (CSG), the macro user try to initiate a hand over request to the femto-cell which is definitely going to fail because the user is not a member of the CSG list [15]. The macro user then experience a strong interference in the downlink channel which lead to a decrease in the Signal-to-Interference plus Noise Ratio (SINR) of the user. Figure 1 describe cross-tier interference in femto-macro heterogeneous network.

\section{SYSTEM MODEL}

A femto-macro Heterogeneous Network (HetNet) where femto cell are deployed in CSG mode is considered. Figure 2 shows the system model, rectangular blocks representing buildings where femto cells are deployed and in between the buildings is the street. Because femto cells are deployed in CSG mode the introduce downlink interference to macro users within the femto coverage. This interference decrease the SNR of the user resulting to a decrease in its throughput

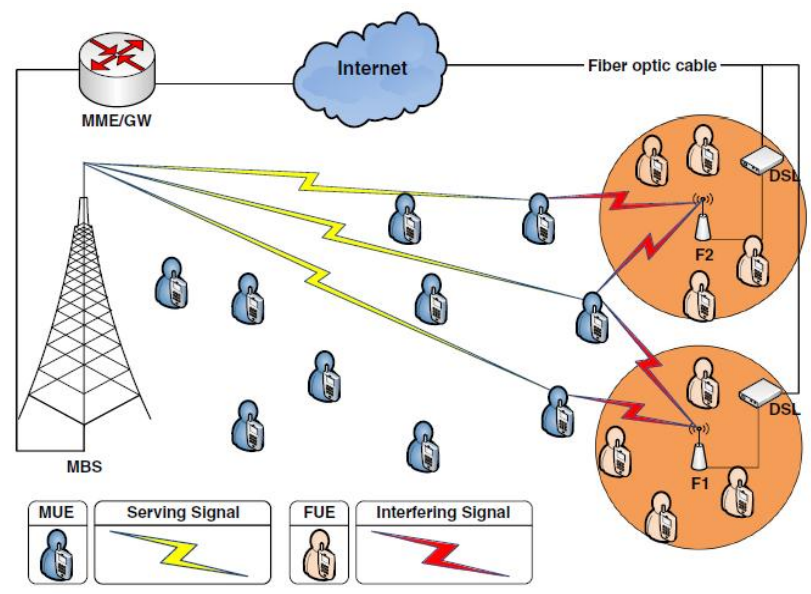

Fig1: Cross-Tier Interference in a Femto-Macro Network [8] 


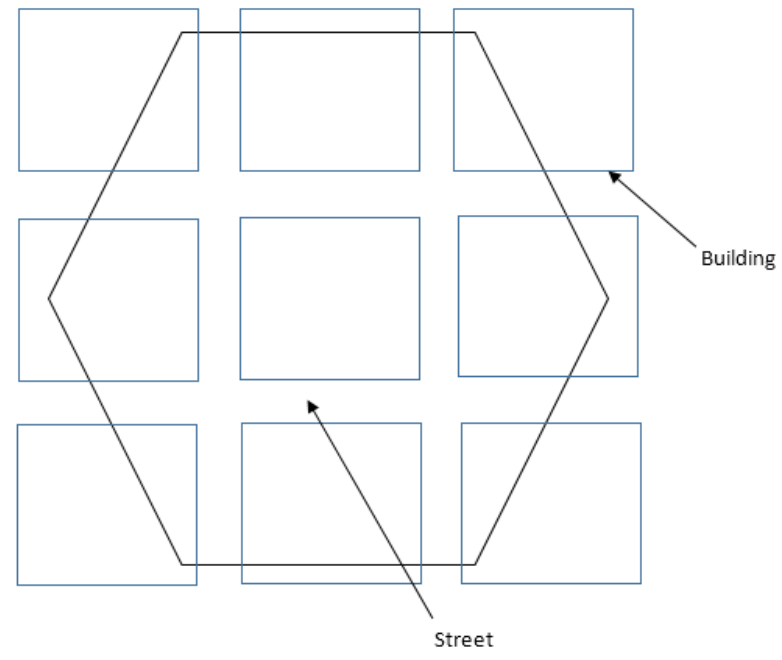

Fig 2: System Model

\subsection{Pathloss Model}

The path loss of the macro and femto user, from their serving eNodeB depends on the position and distance of the user from the macro or femto cell. When the user is inside the building, path loss between the user and the femto cell can be determine from equation [10].

$$
\begin{aligned}
& P L(d B)=38.46+20 \log _{10} R+0.7 d_{2 D, \text {,indoor }}+ \\
& 18.3 n\left(\frac{(n+2)}{(n+1)-0.46}\right)+q * L_{i w}
\end{aligned}
$$

For a femto user outside the building along the street, its path loss from the femto eNodB is given by equation [23].

$$
\begin{aligned}
& P L(d B)=\max \left(15.3+37.6 \log _{10} R, 38.46+\right. \\
& 20 \log _{10} R+0.7 d_{2 D, \text { indoor }}+18.3 n\left(\frac{(n+2)}{(n+1)-0.46}\right)+q * \\
& L_{i w}+L_{o w}
\end{aligned}
$$

For a macro user roaming outside the street, the path loss between the user and the macro cell can be modelled as in [24].

$P L(d B)=15.3+37.6 \log _{10} R$

Where, $R$ and $0.7 d_{2 D \text { indoor }}$

is transmit-recieve distance and penetration loss due to walls inside the appartment respectivly, $n$ is number of penetrated floors, $q$ is number of walls separating apartments between UE and femto cell, $L_{i w}$ is penetration loss of the wall separating apartments, $L_{o w, 1}$ and $L_{o w, 2}$ is the penetration loss of outdoor wall 1 and 2 respectively.

\subsection{SINR Model}

Signal to interference plus noise ratio of a user at any point depends on the interference added by other cells within the network. The SINR of a macro user from the macro cell is modelled as

$$
S I N R_{m, k}=\frac{P_{M, k} G_{m, M, k}}{N_{0} \Delta f+\sum_{M \prime} P_{M \prime, k} G_{m, M I, k}+\sum_{F} P_{F, k} G_{m, F, k}}
$$

Where,

$P_{M, k}$ and $P_{M^{\prime}, k}$ is the transmit power of serving macro-cell $M$ and the neighbouring macro cell $M^{\prime}$ on sub-carrier $k$, respectively.

$G_{m, M, k}$ is the channel gain between macro user $m$ and serving macro cell $M$ on sub-carrierk.

$G_{m, M \prime, k}$ represent the channel gain from neighboring macro-cell.

$P_{F, k}$ is the transmit power of neighbouring femto cell $F$ on sub-carrier $k$.

$G_{m, F, k}$ is the channel gain between macro user $m$ and neighboring femto cell $F$ on sub carrier $k$.

$N_{0}$ is the white noise power spectral density, and $\Delta f$ is sub-carrier spacing.

Similarly, SINR for a femto user can be modelled as:

$$
\operatorname{SINR}_{f, k}=\frac{P_{F, k} G_{f, F, k}}{N_{0} \Delta f+\sum_{M} P_{M, k} G_{f, M, k}+\sum_{F^{\prime}} P_{F^{\prime}, k} G_{f, F^{\prime}, k}}
$$

where:

$P_{F, k}$ and $P_{F \prime, k}$ is the transmit power of serving and neighbouring femto cell on sub-carrier $k$, respectively.

$G_{f, F, k}$ is the channel gain between femto user $f$ and serving femto cell on sub-carrier $k . G_{f, F^{\prime}, k}$ represent the channel gain from neighbouring femto cell.

$P_{M, k}$ is the transmit power of neighbouring macro cell on sub-carrier $k$.

$G_{f, M, k}$ is the channel gain between femto user $f$ and neighbouring macro cell on sub carrier $k$.

$N_{0}$ is the white noise power spectral density, and $\Delta f$ is sub-carrier spacing.

\section{3 Throughput Model}

After determining the SINR and path loss model of a user depending on its environment, next is the determination of it throughput. Throughput of the macro user $m$ and femto user $f$ on a sub-carrier $k$ can be given by [10]:

$C_{m, k}=\Delta f \cdot \log _{2}\left(1+\alpha \operatorname{SINR} R_{m, k}\right)$

(6)

$C_{f, k}=\Delta f \cdot \log _{2}\left(1+\alpha S I N R_{f, k}\right)$

where:

$\alpha$ is a constant for target Bit Error Rate (BER)

BER is set to $10^{-6}$.

$C_{m, k}$ is macro user capacity on a sub-carrier k.

$C_{f, k}$ is femto user capacity on sub carier k.

$\Delta f$ is bandwidth of operation.

$\operatorname{SINR}_{m, k}$ is macro SINR on subcarrier $\mathrm{k}$.

$\operatorname{SINR}_{f, k}$ is femto SINR on subcarrier k.

$\alpha$ is a constant for target bit error rate.

Therefore the total Capacity of serving macro and femto-cell can be express as follows [24]: 


$$
T_{M}=\sum_{m} \sum_{k} \beta_{m, k} C_{m, k}
$$

Similarly for a femto-cell it is given as [25]

$$
T_{M}=\sum_{f} \sum_{k} \beta_{f, k} C_{f, k}
$$

Where $\beta_{f, k}$ and $\beta_{m, k}$ represent subcarriers assignment for macro and femto users respectively. When it is equal to 1 it means the subcarrier is assign otherwise it is equal to zero.

\section{POWER CONTROL}

Power control technique involves increasing and decreasing the transmit power of femto cell to avoid interference caused to nearby users who are not connected to the cell. This interference is mitigated by adjusting the power of femto eNode. The femto eNodeB transmit power is calculated in decibels as [26]:

$P_{f}=\min \left(P_{m}+G_{\theta}-P L_{m}(d)+P L_{f}(r), P_{\max }\right)$

where:

$P L_{f}(r)$ is the line of sight path loss at the target cell radius $\mathrm{r}$.

$P_{m}$ is the transmit power of the macro eNodeB where the femtocell is located.

$P L_{m}(d)$ is the average macrocell pathloss at femtocell distance $\mathrm{d}$.

$G_{\theta}$ is the antenna gain in direction of femto-cell.

$\theta$ is the angle to the femtocell with respect to the sector angle and can be calculated for the case of tree sector site as:

$G_{\theta}=G_{\max }-\min \left[12\left(\frac{\theta}{\beta}\right)^{2}, G_{s}\right]$

where:

$\beta$ is $70 / 180$ the angle where gain pattern is $3 \mathrm{~dB}$ down from peak.

$G_{S}$ is $20 \mathrm{~dB}$ the sidelobe gain level.

$G_{\text {max }}$ is $16 \mathrm{~dB}$ the maximum gain level.

$\theta$ is a range from $-\pi$ to $\pi$

Power control technique can avoid interference completely without degrading its user's capacity or increasing the number of outage only when the interfered users are at the boundary of femto cell. When the interfered users are in close proximity, for example in the same building with the femto eNodeB, the femto eNodeB could adjust its power so much that some of its users will go out of coverage, thereby increasing the number of outage which in turn decreases the total capacity of the system. The flow chart for power control scheme can be seen in fig 3 .

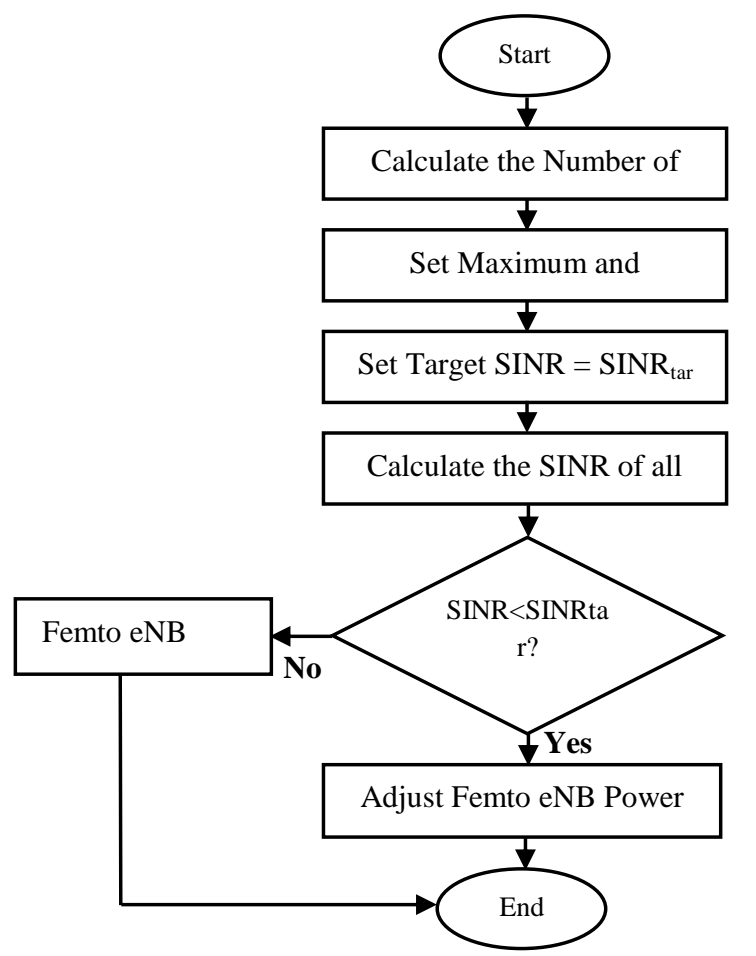

Fig 3: Flow Chart of Power Control eICIC Technique

\subsection{Time Domain}

Time domain scheme for mitigating cross tier interference involve the muting of sub frames by the aggressor eNodeB so that victim UE can be assign during these sub-frames. Time domain technique mitigate interference at the expense of reducing capacity as the eNodeB does not transmit in all subframes. Figure 4 present the flow chart for a time domain eICIC technique.

\subsection{Developed Hybrid Power Control and Time Domain Technique}

The developed hybrid interference mitigation scheme, integrate Power Control (PC) and Time Domain (TD) technique. The hybrid technique mitigate cross-tier interference in a femto-macro HetNet. PC and TD technique when implemented separately, each has its own disadvantage. As in the case of PC technique, mitigating interference becomes a challenge when the victim user is in close proximity to femto eNodeB because it can result to increased percentage outage. While in time domain where interference is mitigated at the expense of reducing the capacity of femto eNodeB since it does not transmit in some sub-frame, especially when the muting ratio is above $50 \%$. In the developed hybrid scheme, a target SINR to be guaranteed to all users and a threshold distance $D_{\min }$ which is referred to as target SINR range is set. For cell edge macro users, that is, users that are at distance above $D_{\min }$ the technique implement power control by reducing and increasing its power adaptively. But for macro users at distance below $D_{\min }$ the technique implements time domain ABS because of the close proximity to the femto eNodeB. The hybrid technique is able to mitigate interference better in comparison to when the techniques are implemented individually and also combines the advantages of both techniques. Fig is a flow chart of the hybrid technique figure 6 . 


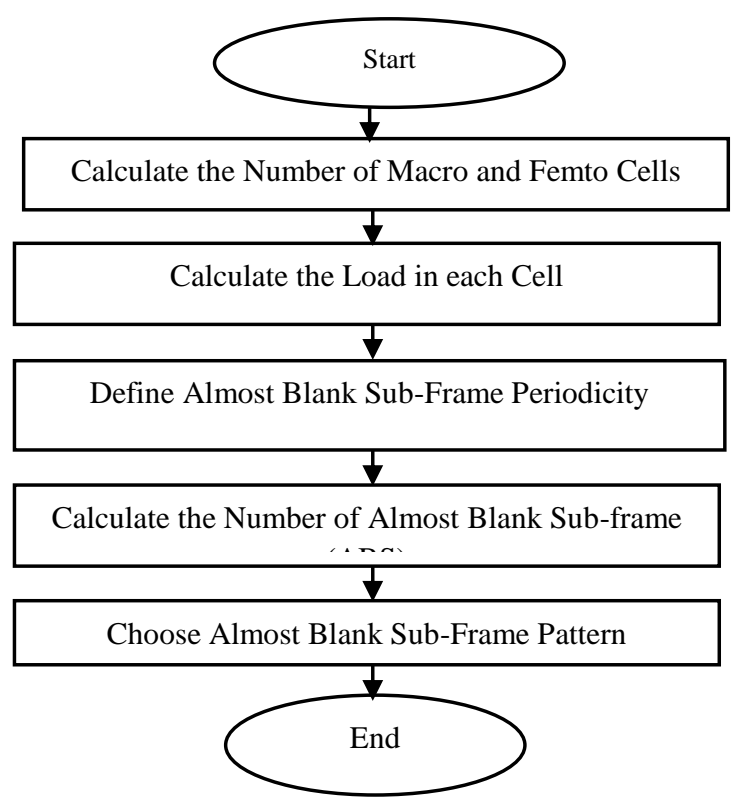

\subsection{Simulation}

The simulation frame work is a Graphical User Interface (GUI) built with MATLAB which the user can interact with by providing simulation parameter. Fig 5 shows the GUI interface, the user interacts with the GUI and provides all the necessary inputs. The path loss of the provided custom set up is calculated depending on the position of the user. The results obtain from the path loss is used to calculate the gain which is used to obtain the SINR of every user. Throughput and sum capacity of the users is calculated from the SINR. The system model topology and configuration depend on the inputs provided by the user at every particular scenario. For the purpose of simulation and result analysis, the configuration of Figure 3.7(a) and 3.7(b) are investigated. Firstly, the model scenario is simulated considering only power control scheme of target SINR after which results is collected for users of interest. Secondly, the model is simulated considering only time domain scheme with different muting ratio and lastly simulation is carried out for the hybrid power control and time domain scheme.

Fig 4: Time Domain eICIC technique flow chart

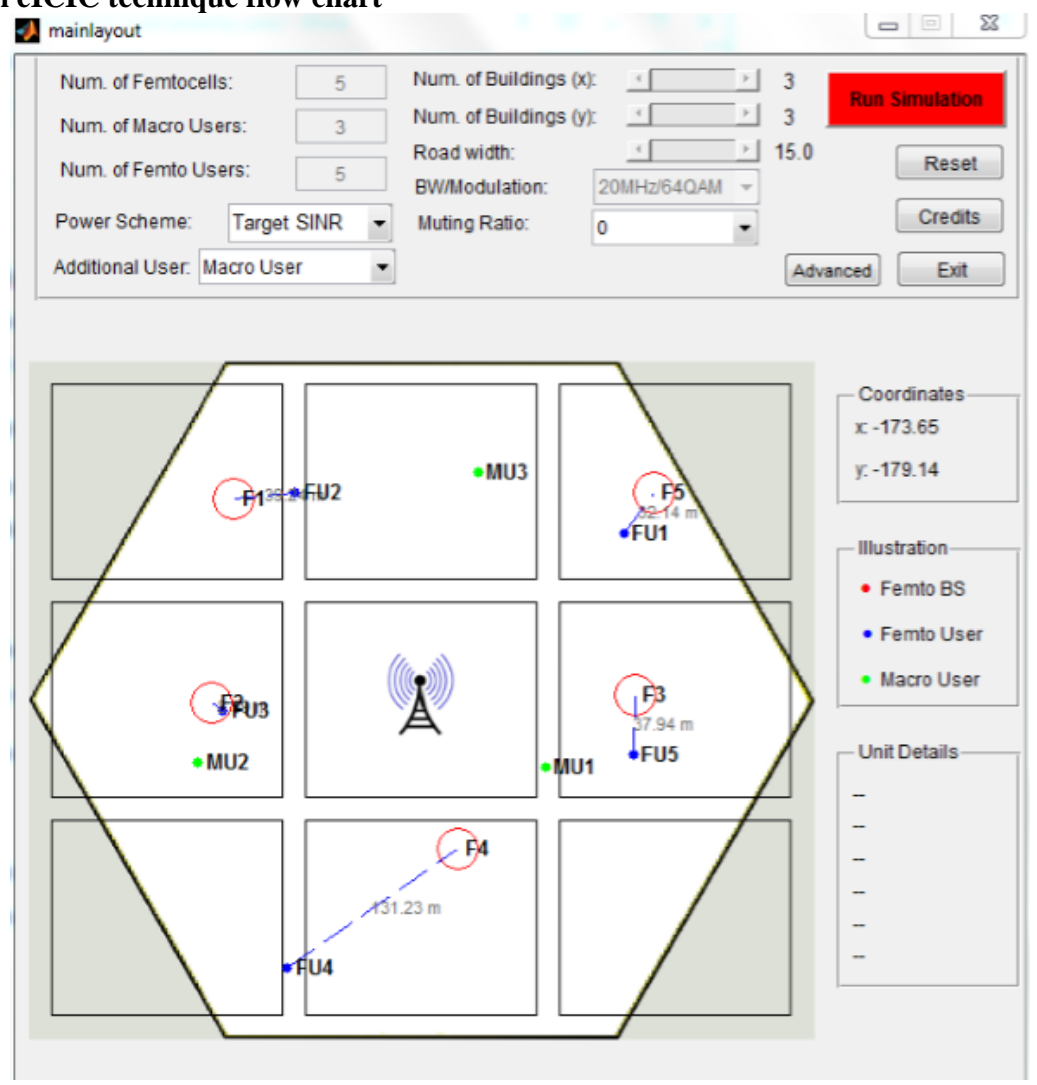

Fig 5: GUI for Simulation

Simulations assumptions and parameters is shown in table 1

\section{RESULTS AND DISCUSSIONS}

In Figure 7, throughput against distance was plotted for a macro user moving towards a femto eNodeB. The plot was obtained by first determining the path loss of the user from equation (3, the path loss depends on the distance of the user from the serving eNodeB. SINR was obtained from equation (4), finally the throughput was obtained from equation (6) and plotted. From the graph of Figure 7 it is seen that as the macro user moves away from the macro eNodeB towards the femto eNodeB its throughput begins to decrease. The femto eNodeB is at 200 meters away from the macro eNodeB, therefore at that point the throughput of the macro user is zero due to interference the macro user experience from the femto eNodeB. 


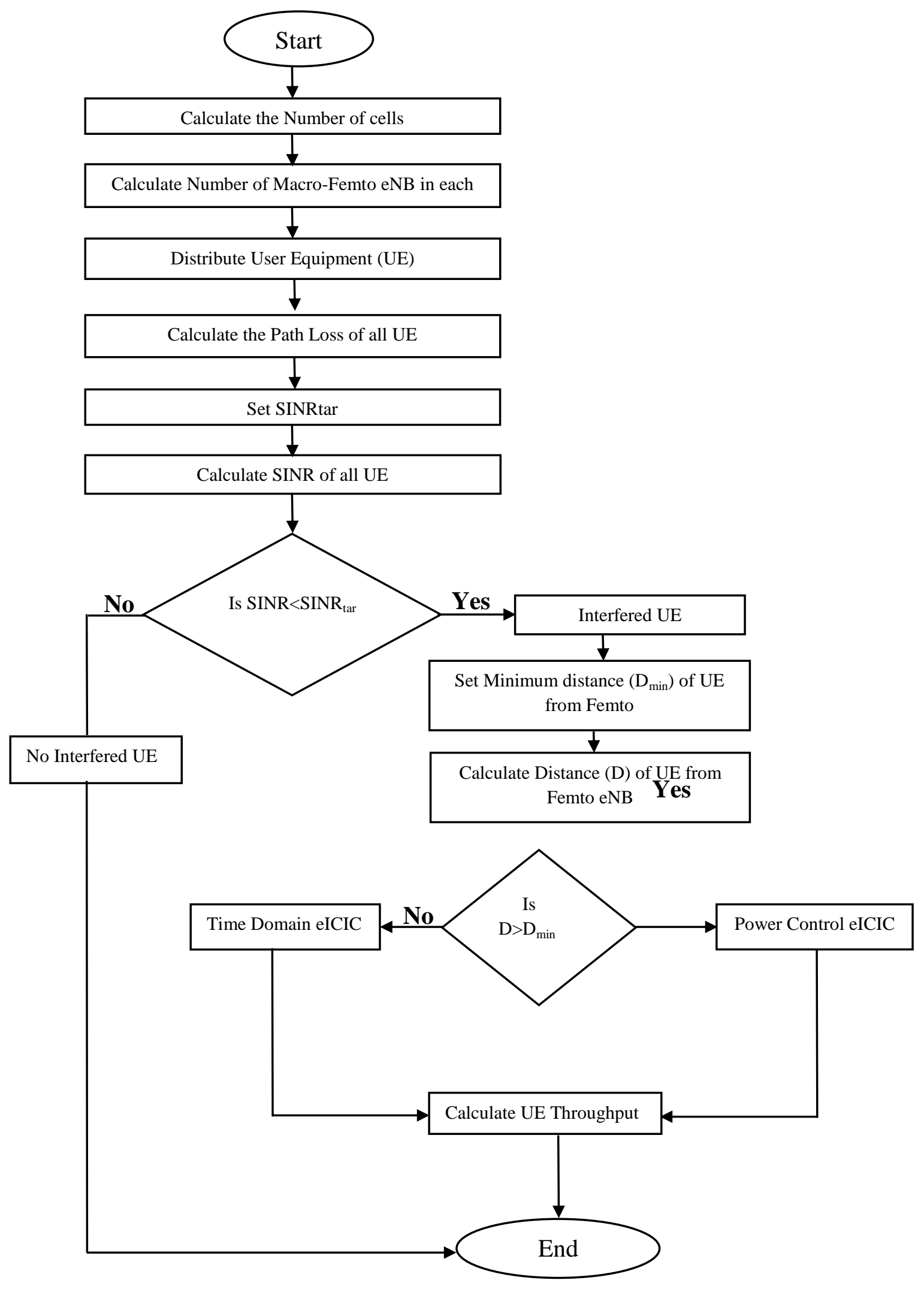

Fig 6: Flow Chart for Hybridized Power Control and Time Domain 
Table 1: System Level Simulation Parameters

\begin{tabular}{|c|c|c|}
\hline Parameters & \multicolumn{2}{|c|}{ Value/Description } \\
\hline Cellular layout & & Single Macrocell \\
\hline Number of Macro eNodeB & & 1 \\
\hline Macrocell radius & & $250 \mathrm{~m}$ \\
\hline Macro eNodeB TX power & & $46 \mathrm{dBm}$ \\
\hline Carrier frequency & & $2 \mathrm{GHz}$ \\
\hline Femto eNodeB max TX pow & wer & $20 \mathrm{dBm}$ \\
\hline Femto eNodeB default TX & & $11 \mathrm{dBm}$ \\
\hline $\mathrm{D}_{\min }$ & \multicolumn{2}{|c|}{$75 \%$ Femto Range } \\
\hline Exterior wall loss & \multicolumn{2}{|c|}{$15 \mathrm{dBm}$} \\
\hline Interior wall loss (low) & \multicolumn{2}{|r|}{$7 \mathrm{dBm}$} \\
\hline Bandwidth (20MHz) & 20 & 15 \\
\hline Modulation type & 64QAM & 16QAM QPSK \\
\hline Subcarrier Spacing & & $15 \mathrm{kHz}$ \\
\hline White noise power density & & $-174 \mathrm{dBm} / \mathrm{Hz}$ \\
\hline Target SINR & & $4 \mathrm{dBm}$ \\
\hline Max. Throughput & & $12 \mathrm{Mbps}$ \\
\hline
\end{tabular}

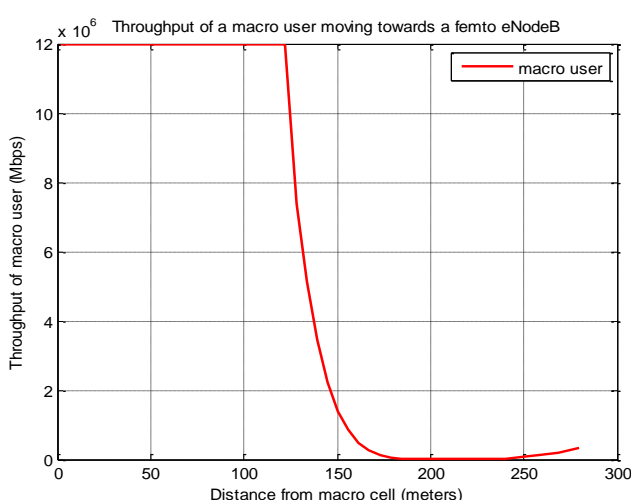

Fig 7: Throughput of Macro User Moving Towards a Femto eNodeB

Figure 8 is a plot of throughput against distance of a femto user moving away from its serving eNodeB. It can be seen that as the user moves away from the serving eNodeB its throughput decreases. However, the decrease is pronounced in the case of femto user because of the size of the cell and high path loss caused by the walls of the building. The decrease in throughput for the case of femto user begins at $23 \mathrm{~m}$, where the throughput is $18 \mathrm{Mbps}$. At $40 \mathrm{~m}$ the throughput has decreases to about $5 \mathrm{Mbps}$, which is about $28 \%$ decrease in an interval of $17 \mathrm{~m}$. Similarly, the throughput of the macro user begin to decrease at $125 \mathrm{~m}$ away from the macro eNodeB with a throughput of $12 \mathrm{Mbps}$. At $150 \mathrm{~m}$ the throughput has decreased to $1.5 \mathrm{Mbps}$, which is about $12.5 \%$ decrease in an interval of $25 \mathrm{~m}$.

Figure 9 is a plot of the total capacity of a femto eNodeB against distance obtained from equation (9) with different muting ratio using time domain scheme. It is seen from the plot that at any particular distance from the femto eNodeB the higher the muting ratio, the lower the total capacity of the femto eNodeB. This is so because the eNodeB does not transmit any data during $\mathrm{ABS}$ frames. At $20 \mathrm{~m}$ distance away from the femto eNodeB the throughput are $0.8 \mathrm{Mbps}, 3.5 \mathrm{Mbps}, 4.2 \mathrm{Mbps}$ and $6.2 \mathrm{Mbps}$ for muting ratio of $0.8,0.5,0.3$ and 0 , respectively. This implies that optimal muting ratio needs to be selected to avoid degrading the femto eNodeB total capacity.

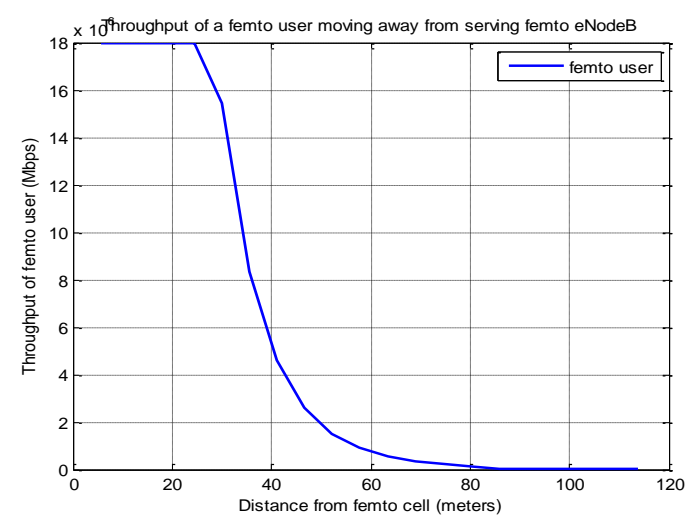

Fig 8: Throughput of a femto user moving away from femto eNodeB

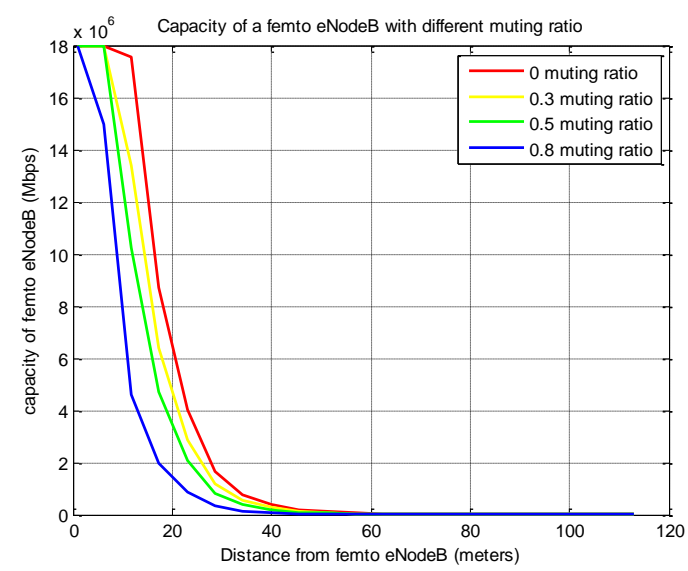

Fig 9: Capacity of Femto eNodeB

Figure 11 is a colour map representing the throughput of a macro user at any point in the macro cell. The result was obtain from the configuration of Figure 10 and colored based on equation [24]. Simulation was carried out for PC and TD with different muting ratio of $0.3,0.5$ and 0.8 . For MU1 the summary of results is shown in Table 2. It can be seen that hybrid techniques with time domain muting ratio of 0.8 gives the highest value of throughput for MU1. This means that the more the increase in the muting ratio at the femto eNodeB the higher the throughput obtained by the macro user. Implementing only TD technique gives lower throughput for muting ratio of 0.3 and 0.5 , but a better throughput is obtain for PC. The result shows that $\mathrm{PC}$ is best used to mitigate interference when the victim user is at the edge of the cell.

For the same configuration of Figure 10 the additional user was changed to Femto User (BS1). This means that the macro cell was coloured based on the throughput of the users connected to femtocell F1 in the configuration. Simulation was also carried out for PC and TD techniques with different muting ratio. The summary of results obtain is shown in Table 3 . From this table it is seen that TD is the best technique that provides FU1 with the best throughput for different muting ratios. 


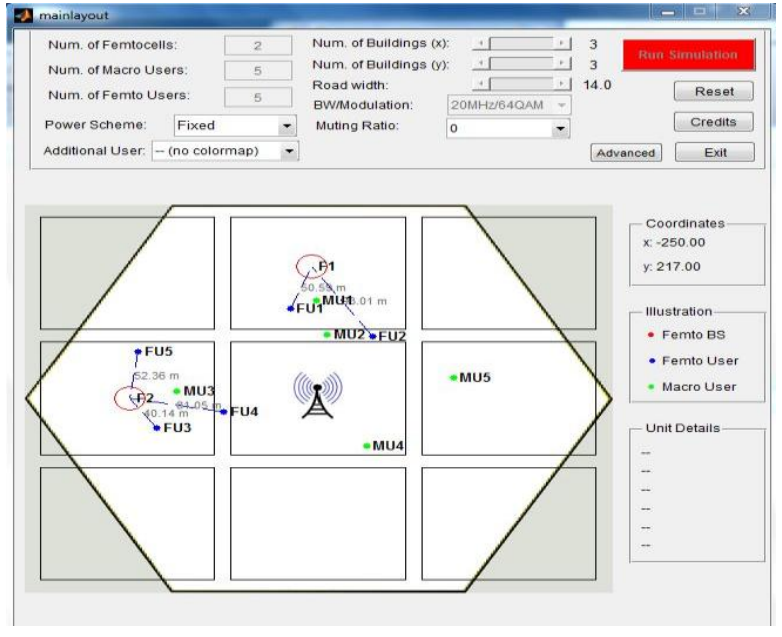

Fig 10: System Model Topology for Analysis

This is because during TD FU1 still has a better coverage from the femto eNodeB only that some sub-frame are muted to serve MU1. PC gives a very low throughput for FU1 because during PC the femto eNodeB has reduced its power to enable it provide the target SINR for the MU1 and as a result leaves FU1 with little or no coverage. This makes PC technique efficient only when the victim users are at the edge of the cell, where adjusting the power of the femto eNodeB make the femto users to experience very low SINR and eventually go out of coverage.

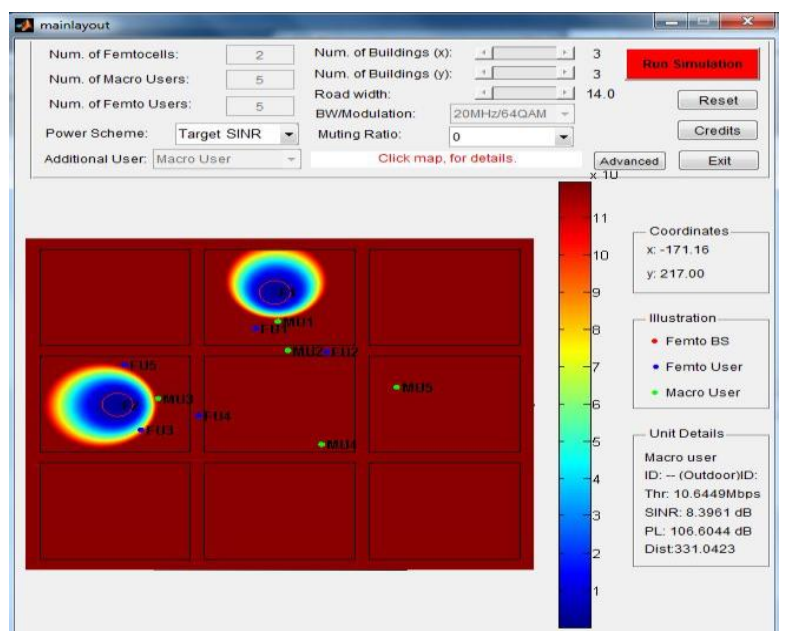

Fig 11: Throughput Colour Map for Implementing PC only

Table 2: Summary of Results obtain for MU1

\begin{tabular}{|l|l|l|l|}
\hline \multicolumn{1}{|c|}{ Parameter } & \multicolumn{1}{|c|}{ PC } & TD & aHPTT \\
\hline Throughput & 10.6449 & 1.0871 & 11.0000 \\
(Mbps) & & & \\
\hline SINR (dB) & 8.3961 & -2.7788 & 9.1048 \\
\hline
\end{tabular}

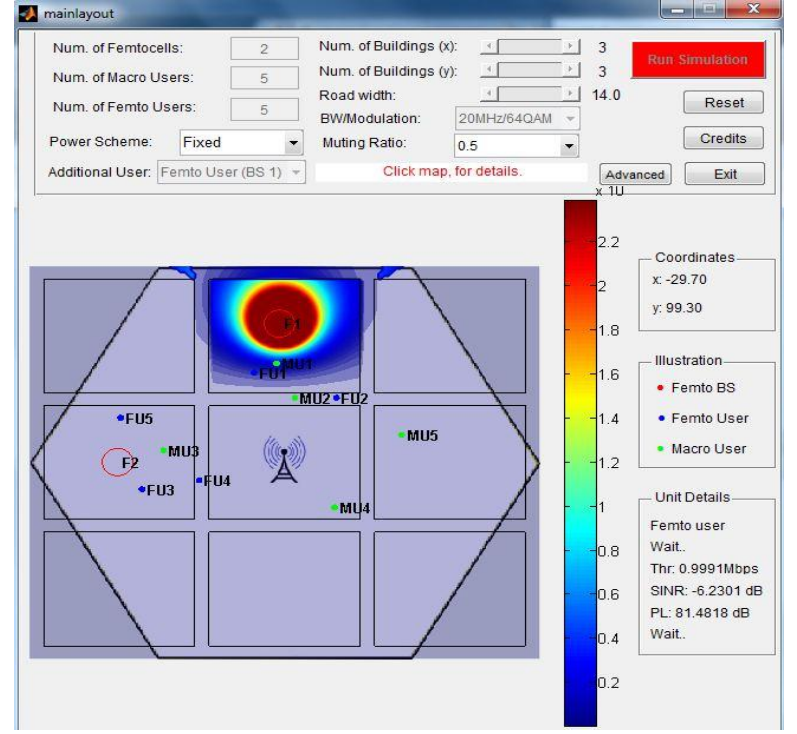

Figure 4.5: Throughput Colour Map for Implementing TD only

Table 2: Summary of Results obtain for FU1

\begin{tabular}{|l|c|l|l|}
\hline Parameter & PC & TD & aHPTT \\
\hline Throughput & 10.6449 & 1.0871 & 11.0000 \\
(Mbps) & & - & 9.1048 \\
SINR (dB) & 8.3961 & 2.7788 & \\
\hline
\end{tabular}

\subsection{Summary of Results}

From the results obtained it can be summarized that hybrid scheme performs better in terms of higher throughput and SINR when compared to implementing either PC or TD only. PC is efficient and performs better only when the victim users are at the edge of the femto eNodeB or when they victim users are passer-by within the vicinity of the femto eNodeB. TD involves muting sub-frames this reduces the capacity of the femto eNodeB, especially at higher muting ratio. TD is efficient when the victim user is not at the edge of the femto eNodeB but closer to femto eNodeB. However, optimal muting ratio need to be selected to avoid degrading the capacity of the femto eNodeB. The Hybrid technique has integrated PC and TD into a single scheme where for victim users at the edge of the femto eNodeB the scheme performs $\mathrm{PC}$ and for victim users who are not at the edge of the cell the scheme performs TD. This is done simultaneously depending on the position of the victim user in the network.

\section{CONCLUSION}

An adaptive hybrid power control and time domain technique to mitigate interference has been proposed and implemented. The technique has integrated PC and TD to mitigate downlink interference as a single technique. The hybrid scheme performs PC and TD simultaneously depending on the position of the victim user in the macro cell. PC was implemented on users who are at the sell edge or passer-by that is for users at distant position greater than $\mathrm{D}_{\min }$. TD was implemented on users who are at close proximity to the femto eNodeB, that is, at a distant 
less than $\mathrm{D}_{\min }$. The aHPTT shows better improvement in terms of total user throughput than PC and TD when implemented separately.

\section{ACKNOWLEDGEMENTS}

The authors are grateful to Etisalat Nigeria, for providing training and sponsoring the Msc in Telecommunication Engineering here at Ahmadu Bello University Zaria.

\section{REFERENCES}

[1] 1. Saquib, N., et al., Interference management in OFDMA femtocell networks: issues and approaches. IEEE Wireless Communications, 2012. 19(3): p. 86-95.

[2] 2. Cisco, Global Mobile Data Traffic Forecast Update, 2015-2020, in Cisco Visual Networking Index. 2016, February 3, 2016. p. 1-39.

[3] 3. Mach, P. and Z. Becvar. Dynamic power control mechanism for femtocells based on the frame utilization. in 2010 6th International Conference on Wireless and Mobile Communications (ICWMC). 2010. IEEE.

[4] 4. Afroz, F., K. Sandrasegaran, and H. Al Kim, Interference Management In Lte Downlink Networks. International Journal of Wireless \& Mobile Networks, 2015. 7(1): p. 91

[5] 5. Cao, F. and Z. Fan. Downlink power control for femtocell networks. in IEEE 77th Vehicular Technology Conference (VTC Spring), 2013. 2013. IEEE.

[6] 6. Saad, S.A., M. Ismail, and R. Nordin, A Survey on Power Control Techniques in Femtocell Networks. Journal of Communications, 2013. 8(12): p. 845-854.

[7] 7. Bartoli, G., et al. Adaptive muting ratio in enhanced Inter-Cell Interference Coordination for LTE-A systems. in International Wireless Communications and Mobile Computing Conference (IWCMC), 2014. 2014. IEEE.

[8] 8. Kurda, R., L. Boukhatem, and M. Kaneko, Femtocell power control methods based on users' context information in two-tier heterogeneous networks. EURASIP Journal on Wireless Communications and Networking, 2015. 2015(1): p. 1-17.

[9] 9. Wang, Y. and K.I. Pedersen. Time and power domain interference management for LTE networks with macrocells and HeNBs. in IEEE Vehicular Technology Conference (VTC Fall), 2011. 2011. IEEE.

[10] 10. Bouras, C., et al., A simulation framework for LTE-A systems with femtocell overlays. Proceedings of the 7th ACM workshop on Performance monitoring and measurement of heterogeneous wireless and wired networks, 2012: p. 85-90.

[11] 11. Lopez-Perez, D., et al., Enhanced intercell interference coordination challenges in heterogeneous networks. IEEE Wireless Communications, 2011. 18(3): p. 22-30.

[12] 12. Pedersen, K.I., et al. Study of Dynamic eICIC in a Realistic Urban Deployment. in IEEE 81st Vehicular Technology Conference (VTC). 2015.
[13] 13. Huang, C., Q. Chen, and L. Tang, Hybrid inter-cell interference management for ultra-dense heterogeneous network in 5G. Science China Information Sciences, 2016: p. 1-13.

[14] 14. Morita, M., Y. Matsunaga, and K. Hamabe. Adaptive power level setting of femtocell base stations for mitigating interference with macrocells. in IEEE 72nd Vehicular Technology Conference Fall (VTC 2010-Fall), 2010. 2010. IEEE.

[15] 15. Zhenwei, W., et al. A novel downlink power control scheme in LTE heterogeneous network. in 2011 International Conference on Computational ProblemSolving (ICCP). 2011. IEEE.

[16] 16. Pang, J., et al. Optimized time-domain resource partitioning for enhanced inter-cell interference coordination in heterogeneous networks. in IEEE Wireless Communications and Networking Conference (WCNC), 2012. 2012. IEEE.

[17] 17. Kshatriya, S.N.S., et al. On interference management based on subframe blanking in heterogeneous LTE networks. in Fifth International Conference on Communication Systems and Networks (COMSNETS), 2013. 2013. IEEE.

[18] 18. Deb, S., et al., Algorithms for enhanced inter-cell interference coordination (eICIC) in LTE HetNets. IEEE/ACM Transactions on Networking (TON), 2014. 22(1): p. 137-150.

[19] 19. Nasri, R., S. Affes, and A. Stephene. Combined Interference Cancellation and Avoidance over the Downlink of Spectrum-Sharing LTE HetNet. in IEEE International Conference on Ubiquitous Wireless Broadband (ICUWB), 2015. 2015. IEEE.

[20] 20. López-Pérez, D., et al., OFDMA femtocells: a roadmap on interference avoidance. IEEE Communications Magazine, 2009. 47(9): p. 41-48.

[21] 21. Palanisamy, P. and S. Nirmala. Downlink interference management in femtocell networks-a comprehensive study and survey. in 2013 International Conference on Information Communication and Embedded Systems (ICICES). 2013. IEEE

[22] 22. Claussen, H., L.T. Ho, and L.G. Samuel, An overview of the femtocell concept. Bell Labs Technical Journal, 2008. 13(1): p. 221-245.

[23] 23. R1-104414, Home enhanced NodeB Power Setting Specifications. 2010: 3rd Generation Partnership Project

[24] 24. TR36.814, Evolved Universal Terrestrial Radio Access (E-UTRA); Further advancements for E-UTRA physical layer aspects 2010, Technical Report 3rd Generation Partnership Project (2010-03): FRANCE.

[25] 25. Lee, S., et al. Interference management in OFDMA femtocell systems using fractional frequency reuse. in International Conference on Communications, Circuits and Systems (ICCCAS), 2010. 2010. IEEE.

[26] 26. Bouras, C., et al., A simulation framework for evaluating interference mitigation techniques in heterogeneous cellular environments. Wireless personal communications, 2014. 77(2): p. 1213-1237. 